\title{
The Effect of Continuous Price Change in the EOQ
}

\author{
E EREL \\ Bilkent University, Ankara, Turkey \\ (Received November 1990; in revised form November 1991)
}

\begin{abstract}
The sensitivity of the basic economic order quantity (EOQ) model to continuous purchase price changes is explored. The phenomenon of continuous price changes exists in several countries and it is not likely to improve. The paper shows that using the conventional EOQ can be quite costly and far from optimal, if the holding cost rate is determined erroneously by ignoring the price change.

Key words -inventory, economic order quantity, sensitivity analysis
\end{abstract}

\section{INTRODUCTION}

THE ECONOMIC order quantity (EOQ) model has been around for about 75 years. The wide popularity of the model is due to several factors, including the ease of teaching, manipulation, and calculation. In addition, the model is highly robust to errors in the specification of the cost parameters and the demand rate, if the holding rate is relatively low. Although the basic model has several quite restrictive assumptions, these assumptions have long been relaxed by new versions that better represent the real world [13]. The objective of this paper is to demonstrate the basic model's sensitivity to continuous purchase price changes, a common situation in highinflation countries (such as Turkey, which had an official average annual inflation rate of $44.3 \%$ during 1982-1989 [1]).

The sensitivity of the model has been examined with respect to changes in the demand rate, setup cost, holding cost [2], and known future price changes [11]. Various extensions of the model were published for situations where the price increase does not coincide with the end of an EOQ cycle [14], and for an optimal policy for the finite and the infinite-horizon problem in which a single change in any or all of the cost factors is allowed [8]. Cost expressions of special order strategy and EOQ strategy for any time horizon of interest have also been developed [14]. Some researchers suggest that the EOQ could be quite sensitive to demand forecast errors when the lead time is nonzero [10]. The effects of advertising, price elasticity and economies of scale, and the possibility of some ordered defective items on the EOQ have been investigated [7], as well as the effect of changing the setup cost in a dynamic lot size model [17]. Woolsey [16] claims that the EOQ model has no sense of reality due to the considerable uncertainty present in the values of the parameters. His closing statement is as follows: "If you continue to love and use the EOQ without knowing what it is costing you, I can only suggest that you deserve each other." In response, Weiss [15] states that the real problem with the basic EOQ model is that it should attempt to manage and change the system rather than optimize the system.

In the studies mentioned above, the planning horizon is usually partitioned into two disjoint time intervals in which a single or some of the cost factors are allowed to differ. This approach is suitable when the inflation rate is stable at low rates or when the cost changes occur infrequently. Models incorporating inflation have been developed $[5,13]$; these studies have 
assumed that the inflation rate is constant and used the present value analysis. The objective of this paper is to examine the effect of continuous purchase price changes on the total cost of the basic EOQ model in a typical year.

In the following section, the basic EOQ model is outlined. In the third section, the effects of purchase price changes on the basic EOQ model are analyzed, and an approximate analytical expression for the optimal order quantity is developed. Finally, in the last section, some concluding remarks on this study are given.

\section{BASIC EOQ MODEL}

The basic EOQ model attempts to identify the order size $Q$ that will minimize the sum of the annual costs of holding and ordering the inventory and the cost of purchasing the inventory. The total annual cost associated with holding, ordering and purchasing the inventory can be expressed as follows:

$$
\mathrm{TC}=D S / Q+Q I C / 2+D C
$$

where $D$ is the annual demand rate, $S$ is the setup (ordering) cost, $Q$ is the order quantity, $I$ is the holding cost rate, and $C$ is the unit purchase cost. Note that the last term in the expression can be neglected since it is a constant; it is included to express the exact value of TC and the effect of a change in $C$ will be examined in the following sections.

Inventory holding cost is the relatively ambiguous cost factor in the model. It includes the opportunity cost of the money invested and the expenses to hold the item in stock and is estimated by management accountants. It is lower bounded by the cost of money to the firm and upper bounded by the best rate of return of the most desired product in an expanding market. The value of $I$ is suggested to be typically in the range $0.15-0.35[6,12]$.

The optimum value of $Q, Q^{*}$, is usually referred to as Wilson's economic lot size and may be expressed as follows:

$$
Q^{*}=(2 D S / I C)^{1 / 2} \text {. }
$$

The TC curve is quite flat in the area of $Q^{*}$ especially if $I$ is relatively small, and this has been recognised quite a while ago $[3,4]$. This implies that reasonably sized deviations from $Q^{*}$ have little impact on TC; for example, if a quantity $Q^{\prime}=(1+p) Q^{*}$ is used instead of $Q^{*}$ where $p$ is the deviation rate from $Q^{*}$, then the percentage cost penalty for not using $Q^{*}$ is $50\left[p^{2} /(1+p)\right]$ [13]. Thus, in most cases inexpensive and crude estimates of the parameters would suffice and certain order quantities may have additional appeal over $Q^{*}$ due to the factors and constraints which are not included in the model [13].

\section{EFFECT OF PURCHASE PRICE CHANGE ON THE BASIC EOQ MODEL}

Suppose the purchase price of the item changes continuously at an annual rate of $p$. In other words, the purchase price of the item $C$ increases to $(1+p) C$ in one year. A nonzero $p$ value implies an increase in TC; the annual holding and purchasing costs would be larger than the ones in the TC expression of the basic model. Note that the annual ordering cost is unaffected. The revised total annual cost expression, $\mathrm{TC}_{p}$, can be written as follows:

$$
\mathrm{TC}_{p}=D S / Q+Q C[1+(Q I / 2 D)]\left[p /\left\{(1+p)^{Q / D}-1\right\}\right] .
$$

The derivation of the above expression is shown in Appendix $\mathrm{A}$. The graph of $\mathrm{TC}_{p}$ as $Q$ increases from zero to $2 D$ is depicted in Fig. 1 for different $p$ values. The other parameters in the figure are as follows: $D=1000, C=1, S=100, I=0.5$.

If an upper bound is imposed on the order quantity, then as depicted in Fig. 1, for $p$ larger than a critical value, $p_{c}$, the upper bound becomes the optimal order quantity. For $p<p_{c}$, both the optimal order quantity, $Q_{p}^{*}$, and the associated optimal total cost, $\mathrm{TC}_{p}\left(Q_{p}^{*}\right)$, increase as $p$ increases. The constraint of imposing an upper bound on the order quantity is a frequently encountered situation in real life; for example, one of the several nonbinding constraints, such as warehouse, finance, etc., turns

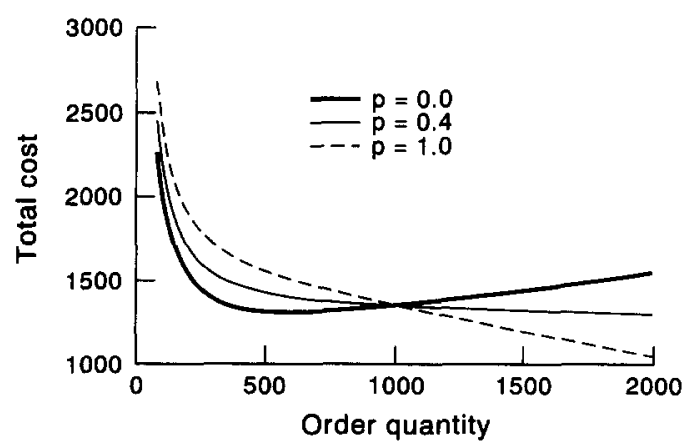

Fig. 1. Total cost values. 
into a binding one as $Q$ increases. The analysis conducted on the value of $p_{c}$ by solving problems with parameters of extreme values suggests that $p_{c}$ is smaller than $I$.

The optimal order quantity, $Q_{p}^{*}$, associated with $p$ could be obtained by setting $\mathrm{dTC}_{p} / \mathrm{d} Q$ equal to zero and solving for $Q$. Unfortunately, the roots of the resulting expression cannot be determined directly. However, an approximate analytical expression for the roots can be derived using the Taylor series expansion of $\mathrm{dTC}_{p} / \mathrm{d} Q$ at $Q=D$. The $\mathrm{dTC}_{p} / \mathrm{d} Q$ expression and the approximate analytical expression of $Q_{p}^{*}$ are given in Appendix $\mathrm{B}$.

The effect of using $Q^{*}$ which ignores the purchase price change can be determined as follows: Let $\mathrm{TC}_{p}\left(Q_{p}^{*}\right)$ denote the cost associated with the optimal order quantity calculated by considering the price change and let $\mathrm{TC}_{p}\left(Q^{*}\right)$ denote the cost associated with $Q^{*}$ calculated by ignoring the price change. Note that the calculation of $\operatorname{TC}_{p}\left(Q^{*}\right)$ involves the price change. The ratio, $\mathrm{TC}_{p}\left(Q_{p}^{*}\right) / \mathrm{TC}_{p}\left(Q^{*}\right)$ measures the sensitivity of the basic model to purchase price changes. $\mathrm{TC}_{p}\left(Q_{p}^{*}\right)$ can be found either by utilizing the approximate analytical expression of $Q_{p}^{*}$ given in Appendix B or by evaluating the $\mathrm{TC}_{p}$ expression for the possible order quantities. Since the analytical expression of $Q_{p}^{*}$ is an approximate one, order quantities from zero to $2 \mathrm{D}$ are considered with increments of $0.02 \mathrm{D}$ in the analysis below.

As $p$ increases, the aforementioned ratio value is expected to decrease, since an increasing $p$ implies increasing annual holding and purchase costs and the increases in these cost terms would cause $Q^{*}$ to deviate from the optimal value. Figure 2 depicts the ratio of $\mathrm{TC}_{p}\left(Q_{p}^{*}\right)$ and $\mathrm{TC}_{p}\left(Q^{*}\right)$ for $p$ values from zero to 1 and $I$ values from 0.2 to 1.2 . The other parameters are

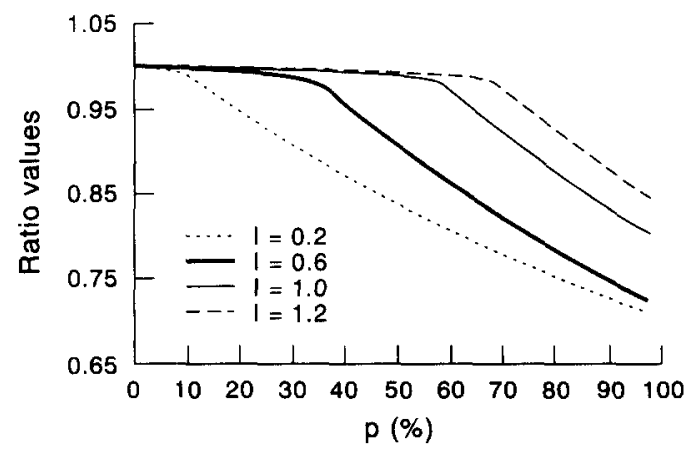

Fig. 2. $\mathrm{TC}_{p}\left(Q_{p}^{*}\right) / \mathrm{TC}_{p}\left(Q^{*}\right)$ values. identical to the ones in Fig. 1. The ratio values are close to unity for small $p$; especially for large $I$, they are close to unity for significantly large $p$. On the other hand, the ratio values are significantly smaller than unity for $p$ larger than $I$. $I$ is expected to be at least equal to or larger than the purchase price change rates, since $I$ includes the opportunity cost which is related to the price changes and the expenses of holding the item in stock. In other words, the portion of the graph in which the ratio values are close to unity is relevant. Thus, determining the value of $I$ with the purchase price change being taken into account diminishes the detrimental effects of a nonzero $p$ on the basic model. On the other hand, an erroneously determined $I$ can result in significantly low ratio values. The ratio values decrease down to $0.71,0.72,0.80,0.84$ for $I$ values of $0.2,0.6,1.0,1.2$, respectively, for $p$ values up to 1 . This suggests that the model with continuous price change is highly sensitive to the errors in the value of $I$.

The model is quite robust to changes in $D$ and $S$. A ceteris paribus analysis conducted to examine the effect of varying $D$ and $S$ on $\mathrm{TC}_{p}\left(Q_{p}^{*}\right) / \mathrm{TC}_{p}\left(Q^{*}\right)$ reveals that the effects are relatively minor.

\section{CONCLUDING REMARKS}

In this article, it is shown that utilizing the basic EOQ model in an environment with continuous purchase price changes can result in significant losses, especially if the important relationship between $p$ and $I$ cannot be established due to erratic price changes. The amount of loss is highly sensitive to $p$ and $I$, for $p$ values larger than $I$, the amount of loss can be of significant size. Fortunately, such cases are quite unrealistic, since the computation of $I$ includes the opportunity cost and the various expenses to hold the item in stock. It is also noted that other parameter values have little effect on the optimal total cost value.

It can be argued that a new EOQ could be calculated at the beginning of each replenishment cycle; thereby, the purchase price change is taken into account. On the other hand, the EOQ is often used as an approximate model for a majority of items (especially $\mathrm{B}$ and $\mathrm{C}$ items which are of medium or low importance) whose policies one does not want to update frequently. 
An approximate analytical expression for the optimal order quantity for an annual change rate of $p$ is also developed by Taylor series expansion. Comparison of the approximate and exact values shows that the difference between the value of one of the roots and the exact value is negligibly small. On the other hand, the other root yields results significantly far from the exact value.

\section{APPENDIX A}

The purchase price increases to $C(1+p)^{(j-1)(Q / D)}$ at the beginning of the $j$ th cycle, since the length of a cycle is $Q / D$. The annual purchase price and the annual holding cost of the item are

$$
Q C \sum_{j=1}^{D / Q}(1+p)^{(j-1)(Q / D)}
$$

and

$$
[Q I C / 2][Q / D] \sum_{j=1}^{D / Q}(1+p)^{(j-1)(Q / D)}
$$

respectively, since there are $D / Q$ cycles in a year. Thus, the revised total cost expression, $\mathrm{TC}_{p}$, can be written as follows:

$$
\begin{aligned}
\mathrm{TC}_{p}=D S / Q+\left[Q^{2} I C / 2 D\right] \sum_{j=1}^{D / Q} & (1+p)^{(j-1)(Q / D)} \\
& +Q C \sum_{j=1}^{D / Q}(1+p)^{(j-1)(Q / D)}
\end{aligned}
$$

The summation

$$
\sum_{j=1}^{D / Q}(1+p)^{(j-1)(Q / D)}
$$

can be written as $\left[p /\left\{(1+p)^{Q / D}-1\right\}\right]$, since

$$
\sum_{n=0}^{N} x^{n}=\left(1-x^{N+1}\right) /(1-x)
$$

for $x(x \neq 0$ or $x \neq 1)$. Thus, $\mathrm{TC}_{p}$ expression can be simplified to the following:

$$
\mathrm{TC}_{p}=D S / Q+Q C[1+(Q I / 2 D)]\left[p /\left\{(1+p)^{Q / D}-1\right\}\right] .
$$

\section{APPENDIX B}

$$
\mathrm{TC}_{p}=D S / Q+Q C[1+(Q I / 2 D)]\left[p /\left\{(1+p)^{Q / D}-1\right\}\right]
$$

Differentiating $\mathrm{TC}_{p}$ with respect to $Q$ results in the following expression:

$$
\begin{aligned}
\mathrm{dTC}_{p} / \mathrm{d} Q= & -D S / Q^{2}+C[1+Q I / D] \\
& \times\left[p /\left\{(1+p)^{Q / D}-1\right\}\right]-Q C[1+Q I / 2 D] \\
& \times\left[\left\{p \ln (1+p)(1+p)^{Q / D}\right\} /\left\{D\left[(1+p)^{Q / D}-1\right]^{2}\right\}\right]
\end{aligned}
$$

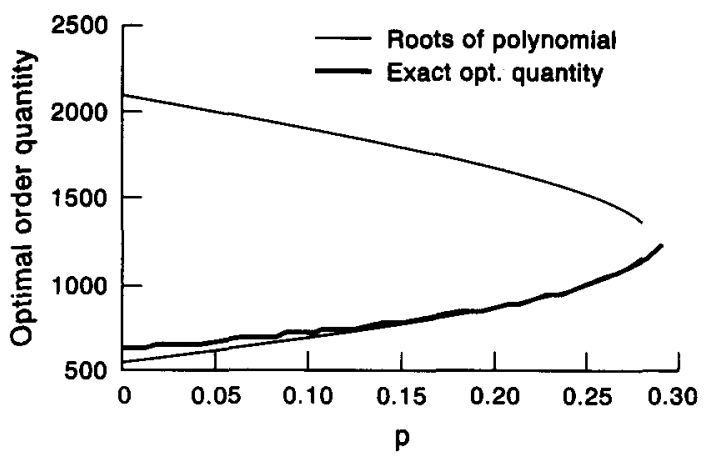

Fig. 3. Exact and approximate values of $Q_{p}^{*}$.

The roots of the polynomial formed by the first three terms of the Taylor series can be found as follows:

$$
\begin{aligned}
Q_{p}^{*}=\left[D f^{\prime \prime}(D)-f^{\prime}(D)\right. \\
\\
\left. \pm\left\{\left[f^{\prime}(D)\right]^{2}-2 f^{\prime \prime}(D) f(D)\right\}^{1 / 2}\right] / f^{\prime \prime}(D)
\end{aligned}
$$

where

$$
\begin{aligned}
f(D)= & -S / D+C[1+I]-C p_{1}[1+I / 2] \\
f^{\prime}(D)= & 2 S / D^{2}+C I / D-\left[2 C p_{1} / D\right][1+I] \\
& +\left[C p_{2} / D\right][1+I / 2] \\
f^{\prime \prime}(D)= & -6 S / D^{3}+\left[3 C p_{2} / D^{2}\right][1+I] \\
& -\left[3 C I p_{1} / D^{2}\right]-\left[C p_{3} / D^{2}\right][1+I / 2]
\end{aligned}
$$

and

$$
\begin{aligned}
& p_{1}=[(1+p) \ln (1+p)] / p \\
& p_{2}=\left[p_{1}(2+p) \ln (1+p)\right] / p \\
& p_{3}=\left[p_{1}\left(p^{2}+6 p+6\right) \ln ^{2}(1+p)\right] / p^{2}
\end{aligned}
$$

Figure 3 depicts the two roots of the polynomial for the problem depicted in Fig. 1. Figure 3 also depicts the exact $Q_{p}^{*}$ values for the same problem. The exact values of $Q_{0}^{*}$ are found by considering order quantities from zero to $2 D$ with increments of $0.02 D$. Note that $p_{c}$ is 0.29 .

\section{REFERENCES}

1. The Central Bank of the Republic of Turkey (1990) Q. Bull. I, 155.

2. Dobson G (1988) Sensitivity of the EOQ model to parameter estimates. Opns Res. 36(4), 570-574.

3. Eilon S (1960) A note on the optimal range. Mgmt Sci. 7(1), 56-61.

4. Eilon S (1963) Elements of Production Planning and Control. Macmillan, New York.

5. Gurnani C (1983) Economic analysis of inventory systems. Int. J. Prodn Res. 21(2), 261-277.

6. Johnson LA and Montgomery DC (1974) Operations Research in Production Planning, Scheduling, and Inventory Control. Wiley, New York. 
7. Lee HL and Rosenblatt MJ (1986) The effects of varying marketing policies and conditions on the economic order quantity. Int. J. Prodn Res. 24(3), 593-598.

8. Lev $B$ and Weiss $H J$ (1990) Inventory models with cost changes. Opns Res. 38(1), 53-63.

9. Markowski EO (1990) Criteria for evaluating purchase quantity decisions in response to future price increases. Eur J. Opns Res. 47(3), 364-370.

10. Mykytka EF and Ramberg JS (1984) On the sensitivity of the EOQ to errors in the forecast of demand. IIE Trans. 16(2), 144-151.

11. Naddor E (1966) Inventory Systems. Wiley, New York.

12. Schonberger RJ (1985) Operations Management, 2nd edn. Business Publications, Texas.

13. Silver EA and Peterson R (1985) Decision Systems for Inventory Management and Production Planning, 2nd edn. Wiley, New York.

14. Taylor SG and Bradley CE (1985) Optimal ordering strategies for announced price increases. Opns Res. 33(2), 312-325.

15. Weiss EN (1990) Lot sizing is dead: Long live lot sizing Prodn Inv. Mgmt J. (1st Quarter), 76-78.

16. Woolsey G (1988) A requiem for the EOQ: An editorial Prodn Inv. Mgmt J. (3rd Quarter), 68-72.

17. Zangwill WI (1987) From EOQ towards ZI. Mgmt Sci. 33(10), 1209-1223.

ADDRESS FOR CORRESPONDENCE: Erdal Erel, Bilkent University, Department of Management, 06533 Bilken,, Ankara, Turkey. 